PII: S0038-1098(96)00369-9

\title{
STUDY OF THE PHOTOLUMINESCENCE INSTABILITY OF POROUS SILICON UNDER LIGHT ILLUMINATION
}

\author{
I.M. Chang, J.C. Fan and Y.F. Chen \\ Physics Department, National Taiwan University, Taipei, Taiwan, Republic of China \\ (Received 4 January 1996; in revised form 24 May 1996 by R.T. Phillips) \\ The profile of photoluminescence instability in porous silicon as a function \\ of time under light illumination was studied. Three stages with different \\ characteristic time scales were identified in the evolution curve. In the first \\ stage, the photoluminescence intensity decays very fast, the second stage is \\ an enhancement process, and finally another slowly decaying stage \\ dominates. A striking behavior was also observed in the evolution profile, \\ which shows that the enhancement stage changes to a decay process when \\ the illumination intensity is increased. With the study of the dependence on \\ the illumination intensity, a qualitative model has been suggested to \\ explain our observations. The effect of oxygen on each evolution stage \\ was also investigated. It gives valuable information about the microscopic \\ structures of porous silicon. Copyright (C) 1996 Elsevier Science Ltd
}

Keywords: A. semiconductor, D. optical properties, E. luminescence.

\section{INTRODUCTION}

The discovery of strong visible photoluminescence (PL) at room temperature from electrochemically etched porous silicon $(\pi-S i)[1,2]$ has resulted in a great deal of attention because of the potential applications in Sibased optoelectronics. Although reports of fairly efficient room temperature luminescence throughout much of the visible spectrum are quite encouraging, instability of luminescence intensity under light illumination has been reported [3-7]. Determining the source of the instability is obviously of critical importance to any potential device application. Systematically fitting of the PL decay profile at a fixed spectroscopic position in vacuum environment has been performed [5]. It is found that the time dependence of the degradation follows a stretched-exponential function which can be attributed to the rate constant in the decay-rate equation being timedependent. In this paper, three distinguishable evolution stages in PL evolution curve are discovered, and three different processes are suggested to be responsible for them respectively, because they have definite time scale and character. All previous reports on time evolution in $\pi$-Si are performed in a relative short time scale compared with our work. Hence, only one or two evolution stages have been observed in the literature. Thus, our new observation may be able to enhance the understanding of the instability behavior in $\pi$-Si. The variations of these three processes are investigated when the illumination intensity is changed suddenly after different history of exposure. A striking phenomenon is found: an enhancement stage changes to a decay stage when the illumination intensity is increased. The role of oxygen is also examined by comparing the evolution with and without atmosphere ambient which provides the information of the corresponding atomic structure in each process. A possible mechanism of the instability is proposed to explain our observation and to give the insights into the physics underlying the luminescence in $\pi-S i$.

\section{EXPERIMENTAL}

The $\pi$-Si specimens were prepared by the electrochemical anodization method. A (100) oriented p-typed wafer with a resistivity of $10-20 \Omega-\mathrm{cm}$ was used. The front side was a polished surface while the back side was an unpolished rough surface. Ohmic contact was made on the back side by evaporating an aluminium thin film and subsequently annealing in $\mathrm{H}_{2}$ environment at $450^{\circ} \mathrm{C}$ for $30 \mathrm{~min}$. The anodization was done under a constant current density of $10 \mathrm{~mA} \mathrm{~cm}^{-2}$ in HF solution 
$\left(\mathrm{HF}: \mathrm{H}_{2} \mathrm{O}: \mathrm{C}_{2} \mathrm{H}_{5} \mathrm{OH}=2: 3: 5\right.$ ) for $2 \mathrm{~min}$. Samples were rinsed in flowing DI water and blown dry by nitrogen gas immediately after electrochemical anodization. The PL experiment was done after several minutes of storage in atmosphere. The behavior of PL is similar for different locations of the same sample, which shows that the sample has a good uniformity. The PL spectrum is reproducible, if the sample were prepared under the same conditions. The aged effect was negligible on the time scale of the experiment. Thus, the condition of the nanostructure on different parts of the same sample and on different samples prepared under the same condition is assumed to be the same at the start of each measurement. The PL spectra were measured using a SPEX $0.85 \mathrm{~m}$ double spectrometer, and a photomultiplier tube. The excitation source was an Argon ion laser with excitation wavelength of $488 \mathrm{~nm}$ focused on a spot with the radius about $300 \mu \mathrm{m}$. The Fourier transform infrared (FTIR) absorption measurements were made on Nicolet ZDS FT-IR spectrometer at room temperature.

\section{RESULTS AND DISCUSSION}

A plot of PL intensity vs exposure time is shown in Fig. 1(a) with the sample exposed to an argon ion laser

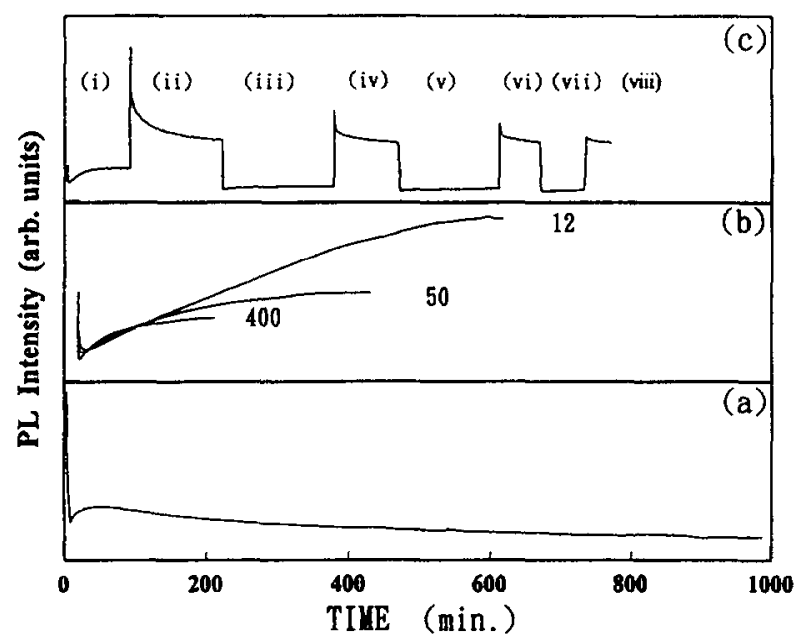

Fig. 1. (a) Time dependence of photoluminescence intensity at $14500 \mathrm{~cm}^{-1}$ for II-silicon irradiated by continuous $\mathrm{Ar}^{+}$laser light of $200 \mathrm{~W} \mathrm{~cm}^{-2}$. (b) Time dependence of photoluminescence intensity at $14500 \mathrm{~cm}^{-1}$ under irradiation by continuous $\mathrm{Ar}^{+}$laser light with different power densities given in units of $W \mathrm{~cm}^{-2}$. The initial PL intensity is normalized to the same value. Each measurement was taken on a different spot of the same sample. (c) Kinetics of the photoluminescence measured at $14500 \mathrm{~cm}^{-1}$. The excitation intensity is $10 \mathrm{~W} \mathrm{~cm}^{-2}$ for (i), (iii), (v) and (vii), and $50 \mathrm{~W} \mathrm{~cm}$ for (ii), (iv), (vi) and (viii), while the same sample point is maintained. beam of power density $200 \mathrm{~W} \mathrm{~cm}^{-2}$ in air. The spectrometer was fixed at $14500 \mathrm{~cm}^{-1}$, near the maximum emission position of the PL spectrum for that sample. The peak position and the line shape of the PL spectrum roughly remain fixed during the experiment. Three evolution stages can be identified in this evolution curve. In the beginning, the PL signal decays very fast toward a lower emission level, as is usually reported. The short time scale of this process is described as $\tau_{s}$. At the same time, another enhancing process is acting. The $\mathrm{PL}$ increases gradually, when the initially decaying process is nearly finished, with an intermediate time scale $\tau_{m}$. This growing stage proceeds until another slowly decaying process dominates. This finally decaying stage has a time scale denoted as $\tau_{l}$. In general, the phenomenon of the growing stage is more pronounced for the samples prepared under a higher HF concentration or a lower electrochemical current density. The relation of $\tau_{l} \gg \tau_{m} \gg \tau_{s}$ holds for all samples prepared at different electrochemical conditions and exposed under different excitation intensities. According to Fig. 1(a), the ranges of the typical values of $\tau_{l}, \tau_{m}$ and $\tau_{s}$ are $500 \mathrm{~min}, 50 \mathrm{~min}$ and $5 \mathrm{~min}$, respectively.

To obtain more information, the evolution was measured at different illumination intensity. The results are shown in Fig. 1(b). The numbers indicate laser power densities in units of $\mathrm{W} \mathrm{cm}^{-2}$, and the initial PL intensity is normalized to the same value. The PL intensity was monitored at the same wavelength as taken above. Each measurement was taken on a different spot of the same sample. Several trends with increasing illumination intensity can evidently be seen, which indicate the photoassistant character of these evolution processes. First, the rates of PL decay and enhancement are faster. That is, the time scales are shorter under stronger illumination. Second, the minimum PL intensity of the valley between the initial decay and growing stage is lowered with increasing illumination intensity. Third, the peak intensity of PL emission before the dominance of the $\tau_{l}$ decay process, is lower for stronger illumination.

In addition to the above measurements of constant intensity exposure, the PL evolution profile with the illumination source interrupted by different intensity and periods have also been studied. The results are shown in Fig. 1(c). The exposure intensity of 10 or $50 \mathrm{~W} \mathrm{~cm}^{-2}$ was used alternatively in Fig. 1(c), with the former for intervals labeled (i), (iii), (v) and (vii), and the latter for those labeled (ii), (iv), (vi) and (viii). All the time intervals between the alternative illumination intensity in Fig. 1(c) lasted for about $\tau_{m}$. It is long enough to exhibit the as mentioned $\tau_{m}$ time scale PL. variation and short enough to avoid the effect of $\tau_{l}$ time decay. In the interval (i) of Fig. 1(c) the PL intensity decays very fast initially and then proceeds with a 
growing stage. However, the PL signal changes to a decay process with time scale $\tau_{m}$ when the excitation is suddenly increased to $50 \mathrm{~W} \mathrm{~cm}^{-2}$, as shown in the interval (ii). After a period of exposure by stronger intensity, the PL rises with time of the illumination intensity is changed back to the lower one. To obtain the enhancement (decay) of PL after sudden decrease (increase) of excitation intensity, two conditions are necessary: (I) large variation in excitation intensity, (II) a prolonged exposure in the previous period lasts for a time scale of about $\tau_{m}$. It is worth considering that the growth (decrease) of PL may follow the decrease (growth) of local temperature after varying the external light source. However, further experiments show that the evolution is irreversible in the dark, we thus can rule out the possibility of laser heating. Variation similar to those of interval (i) and (ii) could be repeated many times as we alternate the excitation intensity, as shown in Fig. 1(c). However, the PL intensity in the latter repetition is lower, and the overall contour in Fig. 1(c) decays with a time scale about $\tau_{l}$. This implies that it may have the same origin as that of the finally $\tau_{l}$ decay process in Fig. 1(a).

Let us now consider a possible model to explain the above observations. We propose that transitions between some specific centers, which have different effects on the light emitting property of $\pi-\mathrm{Si}$, are responsible for the various behaviors of the PL evolution. Even though the exact mechanism of the PL emission in $\pi$-Si is not well established, a sufficient diversity of surface-chemistry related PL results [4-8] indicates that several light emitting centers such as $\mathrm{Si}-\mathrm{H}$ and $\mathrm{Si}-\mathrm{O}-\mathrm{H}$ complexes and nonradiative recombination centers such as dangling bonds exist on the surface of $\pi-\mathrm{Si}$. The structure of these centers and their effect on the property of PL may be changed due to the injection of photons, similar to observations in amorphous semiconductors [8]. Four kinds of center $A, B, C$ and $D$ are suggested to be responsible for the three processes with different time scales mentioned above. The transitions involved can be understood with the assistance of the four-level configuration diagram shown in Fig. $2[9,10]$. The vertical axis shows the relative free-energy change in the transitions. The horizontal axis is a one-dimensional; representation of a multidimensional configuration space for a specific defect site. The valleys in the energy curves represent the metastable states for these centers. The serial relation of center B, C and D is taken to explain the anomalous $\tau_{m}$ growth and decay phenomenon in Fig. 1(c). Simply, the story of the time scale $\tau_{s}$ is caused by the transition of center A to B. The microscopic structure of the corresponding centers will be discussed later. The barriers to the transition between centers are along the most easily attainable route, through which the defects passes during transitions. Disorder produces a variation in barrier heights and energy minima among different defect sites, in an amorphous material as well as in $\pi$-Si. The ground-state energy and the metastable minimum can also vary among the different defects. Each individual defect, however, has a well-defined energy curve. The initial decay with time scale $\tau_{s}$ is the result of transitions of some center $\mathrm{A}$ in $\pi$-Si to center $\mathrm{B}$, because the $\mathrm{B}$ center is a more effective non-radiative recombination center than A, as our microscopic model states. Transition from center $\mathrm{B}$ to $\mathrm{C}$ is responsible for the growing process. In our microscopic model, this is due to the passivation of an efficient non-radiative recombination center $B$ to a less effective center $C$. We thus can avoid employing a new light emitting center, because no experimental evidence is given for the existence of two different emission origins. The transition from center $C$ to the most stable center $D$ causes the finally decay process. The transitions corresponding to $\tau_{s}$ and $\tau_{m}$ require the illumination of light to overcome energy barriers and become faster for stronger illumination as shown in Fig. 1(b).

A macroscopic averaged time constant for all the three kinds of transition is employed in the following discussion, although the energy barriers (and time constants) vary at different defect sites of the same kind in our samples, as mentioned in the last paragraph [5]. Such a simplification is sufficient to produce all the qualitative phenomena we observed. The transition probabilities of $\mathrm{B}$ to $\mathrm{A}, \mathrm{C}$ to $\mathrm{B}$, and $\mathrm{D}$ to $\mathrm{C}$ are postulated to be negligible, because the energy barriers are expected to be much higher than that of the corresponding transitions in the opposite direction. The $n_{A}, n_{B}, n_{C}$ express the number of centers for the respective states per unit volume. The rate

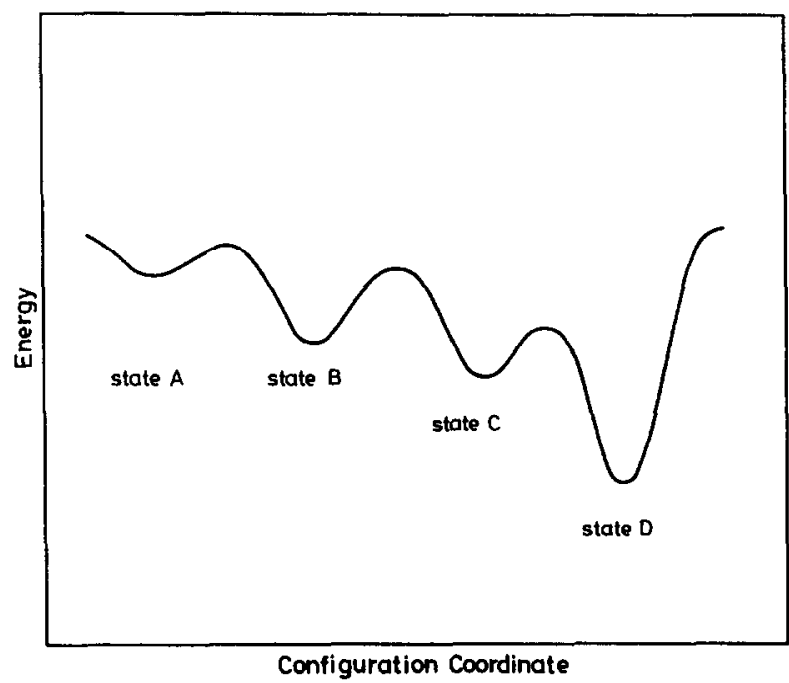

Fig. 2. Energy-configuration diagram used for a phenomenological description of the metastable states in porous silicon. Further descriptions are in the text. 
equations are:

$$
\left\{\begin{array}{l}
\dot{n}_{A}=-\frac{1}{\tau_{A B}} n A \\
\dot{n}_{B}=\frac{1}{\tau_{A B}} n_{A}-\frac{1}{\tau_{B C}} n_{B}, \\
\dot{n}_{C}=\frac{1}{\tau_{B C}} n_{B}-\frac{1}{\tau_{C D}} n_{C}, \\
\dot{n}_{D}=\frac{1}{\tau_{C D}} n_{C} .
\end{array}\right.
$$

$\tau_{A B}, \tau_{B C}, \tau_{C D}$ are the averaged time constant of the transitions from state $\mathrm{A}$ to $\mathrm{B}, \mathrm{B}$ to $\mathrm{C}$ and $\mathrm{C}$ to $\mathrm{D}$, respectively. Intuitively, it seems obvious to take $\tau_{A B}=\tau_{s}, \quad \tau_{B C}=\tau_{m}$, and $\tau_{C D}=\tau_{l}$. This assignment implies that no observable evolution process of time scale $\tau_{m}$ would reappear once the sample has been illuminated for a time longer than $\tau_{m}$. However, this result is contrasted with the observation in Fig. 1(c) where the evolution of time scale $\tau_{m}$ reappears. Thus a longer time scale is expected for the transition of center $B$ to $\mathrm{C}$, this should be $\tau_{l}$, and the observation of $\tau_{l}$ being the time scale of the contour in Fig. 1(c) may further support this attribution. In addition, the decay process of the transition from center $C$ to $D$ measured in vacuum has a time scale of $\tau_{m}$ as discussed below. The assignment of $\tau_{A B}=\tau_{s}, \tau_{B C}=\tau_{l}$, and $\tau_{C D}=\tau_{m}$ is therefore considered. The exact solution of equation (1) is then

$$
\left\{\begin{aligned}
n_{A}(t)= & n_{A}(0) \mathrm{e}^{-t / \tau_{s}} \\
n_{B}(t)= & n_{B a} \mathrm{e}^{-t / \tau_{s}}+\left[n_{B}(0)-n_{B a}\right] \mathrm{e}^{-t / \tau_{l}}, \\
& n_{B a}=\frac{1 / \tau_{s}}{1 / \tau_{l}-1 / \tau_{s}} n_{A}(0) \approx-n_{A}(0) \\
& \times\left[1+o\left(\tau_{s} / \tau_{l}\right)\right] \\
n_{C}(t)= & n_{C a} \mathrm{e}^{-t / \tau_{s}}+n_{C b} \mathrm{e}^{-t / \tau_{l}} \\
& +\left[n_{C}(0)-n_{C a}-n_{C b}\right] \mathrm{e}^{-t / \tau_{m}} \\
& n_{C a}=\frac{1 / \tau_{l}}{1 / \tau_{m}-1 / \tau_{s}} n_{B a} \approx 0+o\left[\left(\tau_{s} / \tau_{l}\right) n_{A}(0)\right] \\
& n_{C b}=\frac{1 / \tau_{l}}{1 / \tau_{m}-1 / \tau_{l}}\left[n_{B}(0)-n_{B a}\right] \\
& \sim \frac{\tau_{m}}{\tau_{t}}\left[n_{B}(0)+n_{A}(0)\right]\left[1+o\left(\tau_{m} / \tau_{l}\right)\right] \\
n_{D}(t)= & n_{D}(0)+\left[n_{A}(0)-n_{A}(t)\right]+\left[n_{B}(0)-n_{B}(t)\right] \\
& +\left[n_{C}(0)-n_{C}(t)\right] .
\end{aligned}\right.
$$

$n_{A}(0), n_{B}(0), n_{C}(0)$ and $n_{D}(0)$ are the initial concentrations of center $A, B, C$, and $D$, respectively. The initial decay of time constant $\tau_{s}$ in Fig. 1 is thus described by the time function of center $n_{A}$ or $n_{B}$. The increasing of $n_{C}$, from the transition of center $B$, causes the enhancement of PL emission, as stated in the above paragraph. The decreasing of $n_{C}$, from transferring to center $\mathrm{D}$, makes the final PL decay. Thus, the magnitude of $n_{C}$ is conveniently taken to characterize the intensity of the PL evolution qualitatively when $t>\tau_{s}$, for larger $n_{C}$ corresponding to stronger PL emission. The explanation of the PL growth thus lies in the capability of the accumulation of $n_{C}$. Figure $1 \mathrm{a}$ is therefore explained.

The observation of Fig. 1(c) would be discussed next. The solution of $n_{C}$ contains two parts when $t>\tau_{s}$. The first term is viewed as a quasi equilibrium concentration $n_{C e}=n_{C b} \mathrm{e}^{-t / \tau_{l}}$ when the measuring time is shorter than $\tau_{l}$. The other term makes the $n_{C}$ approach to this quasi equilibrium concentration in a time $\tau_{m}$. The time constants $\tau$ in $n_{C e}$ vary with excitation intensity, so does the quasi equilibrium concentration. The time constant $\tau_{s}$ and $\tau_{m}$ become shorter on stronger illumination intensity, as mentioned in the analysis of Fig. 1(b), The $n_{B}$ will decay with a new time constant for $t_{s}<t$ when the illumination intensity is changed. According to equation (2), the quasi equilibrium concentration is about equal to $\tau_{m} / \tau_{l}$ times $n_{B}(t)$ when $t>\tau_{s}$, no matter how the history of illumination is. This quasi equilibrium concentration becomes smaller when excitation intensity is increased, if the dependence of illumination intensity on $\tau_{m}$ is stronger than that of $\tau_{l}$. This assumption can readily be seen from Fig. 1(b). The reduction of the maximum attainable PL emission with increasing excitation intensity when $t>\tau_{s}$ in Fig. 1(b) is consistent with the decreasing in the quasi equilibrium concentration of $n_{C_{e}}$ with increasing illumination. The decay in interval (ii) of Fig. 1(c) therefore can be understood as the developing of $n_{C}$ to a new lower quasi equilibrium concentration. The enhancement in interval (iii) of Fig. 1(c) is the result of the accumulation of $n_{C}$ from this new level to its original equilibrium concentration in interval (i) of Fig. 1(c). The growth and decay process can be repeated many times as long as the measurement is performed within a time scale $\tau_{l}$, and thus the behavior of Fig. 1(c) is explained.

The effect of atmosphere was studied by evacuating the sample space during the PL evolution experiment. Figure 3(a) displays the time function of PL signal, with the period of (ii) taken in vacuum environment and the others [(i) and (iii)] in air. Throughout Fig. 3(a), continuous illumination of $20 \mathrm{~W} \mathrm{~cm}^{-2}$ at the sample spot is maintained and the PL signal is measured at the same energy. Behavior in the period (i) of Fig. 3(b) is normally, where the PL signal decays initially and then become enhanced. However, the enhancement disappears when the sample space is evacuated. A decay with time scale $\tau_{m}$ presents instead, as shown in period (ii) of Fig. 3(b). The enhancement of time scale $\tau_{m}$ reappears, in the period of (iii) after the injection of air. The implication of Fig. 3(a) is straightforward within the base of our model. The transition from center $B$ to $C$ is forbidden without air and only the transition from $C$ to $D$ is available then. The behavior of pure $C$ to $D$ transition is just the observed $\tau_{m}$ decay. Figure $3(a)$ is thus 


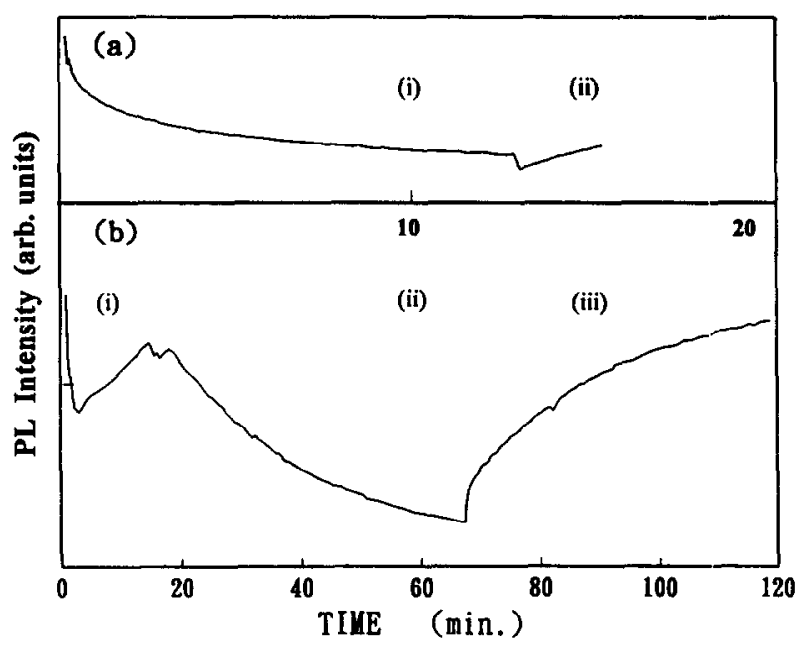

Fig. 3. (a) Time dependence of photoluminescence intensity at $14500 \mathrm{~cm}^{-1}$ irradiated by continuous laser light of $20 \mathrm{~W} \mathrm{~cm}^{-2}$. Both regions (i) and (iii) were performed in air, and (ii) was taken in vacuum while the same sample point is maintained. (b) Time dependence of photoluminescence intensity at $14500 \mathrm{~cm}^{-1}$ irradiated by continuous laser light of $20 \mathrm{~W} \mathrm{~cm}^{-2}$. The region (i) was done on an evacuated chamber and (ii) is measured in air while the same sample point was maintained.

explained. Period (i) of Fig. 3(b) shows the PL intensity as a function of time in an evacuated vacuum chamber, the illumination intensity and the monitor position are the same as that in Fig. 3(a). The presence of $\tau_{m}$ decay is similar to that in period (ii) of Fig. 3a. The transition from state $A$ to $B$ is thus forbidden in vacuum, as indicated in the absence of $\tau_{s}$ decay in period (i) of Fig. 3(b). In the period of (ii), atmosphere was introduced while the same sample point is maintained. The fast $\tau_{s}$ decay in Fig. 3(b) (ii) and the relative factor of reduction during this $\tau_{s}$ decay is close to that in period (i) of Fig. 3(a). The lack of the $\tau_{s}$ decay in period (iii) of Fig. 3(a) is because most of the center $A$ has transferred to center $B$ in the period (i) of Fig. 3(a). This is consistent with the observation of Tischler et al. [4], where a dramatic reduction in $\mathrm{PL}$ intensity has been observed after the introduction of oxygen. The role of oxygen is also demonstrated in our FTIR measurement. Figure 4(a) represents the FTIR measurement of the as-anodized sample. It contains mainly the hydrogen related component. Figure 4(b) is the spectrum of the same sample after prolonged Argon ion laser illumination in air. The hydrogen related component is reduced and the oxygen related component is increased after illumination.

Based on the above measurement, the corresponding microstructures of center $\mathrm{A}, \mathrm{B}, \mathrm{C}$ and $\mathrm{D}$ may be constructed. Because the transition from center $A$ to $B$ is forbidden without air, this process may be the breaking of weak $\mathrm{Si}-\mathrm{Si}$ bonds (center $\mathrm{A}$ ) due to the insertion of oxygen [4]. This process creates dangling bonds (center B) which serve as the nonradiative recombination centers and cause the reduction of PL efficiency. The transition from state $B$ to $C$ also requires the participation of oxygen and its proceeds with the long time scale $\tau_{l}$.

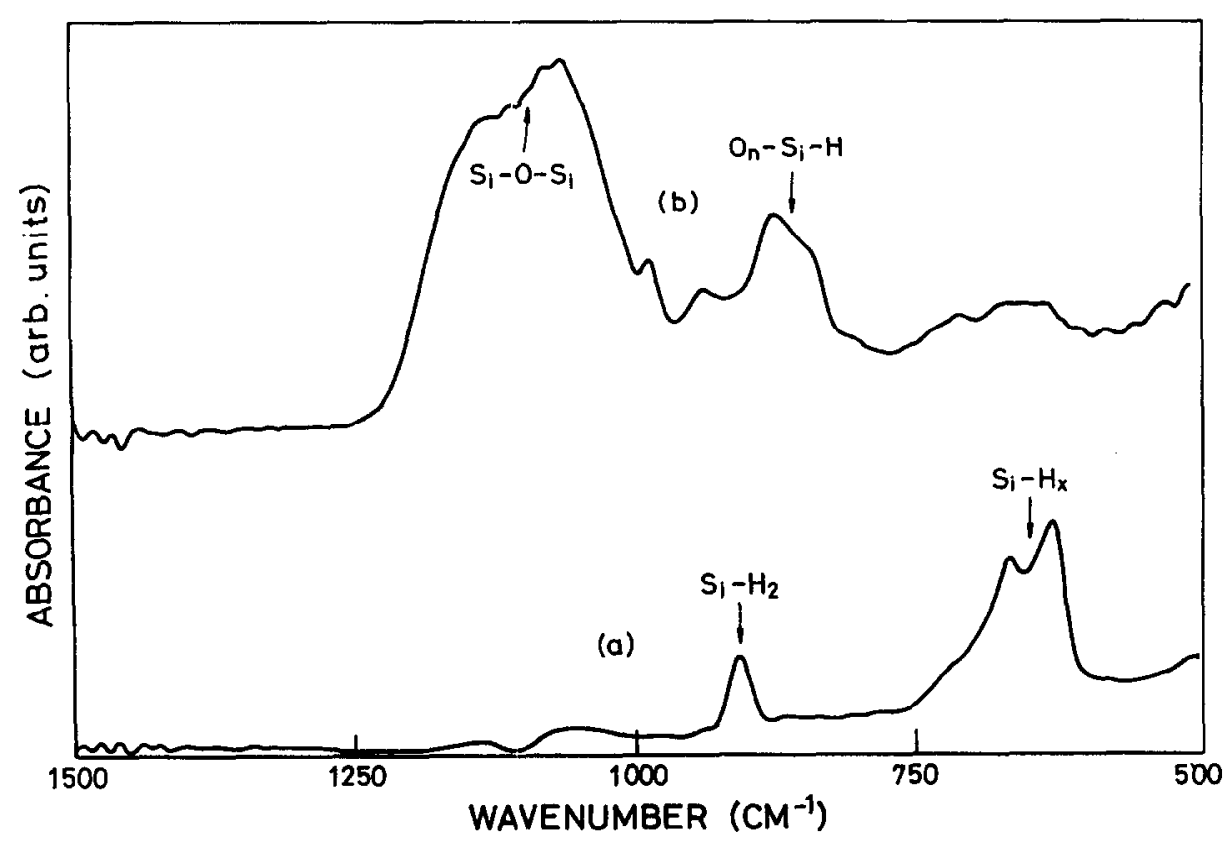

Fig. 4. Infrared absorption spectra of (a) the as-anodized sample and (b) the same sample after prolonged illumination in air. 
We therefore suggest that it involves the diffusion of oxygen to a metastable center $C$, which reduces the number of dangling bonds, and hence increases the PL signal $[4,7]$. The relaxation of this metastable center to the most stable center $D$ with the creation of dangling bonds may be attributed to the cause of the final decay process with time scale $\tau_{m}$.

\section{CONCLUSIONS}

We have observed three distinguishable stages in porous silicon under light exposure. An anomalous behavior of the PL evolution profile has also been obtained when the illumination intensity is changed to a different value. The important role of the oxygen involved in the evolution process has been evidenced by comparing the PL evolution experiment in vacuum and in air. A microscopic model has been proposed to explain our observations. Further studies based on our work may be able to enhance the understanding of the light emitting mechanism in porous silicon.
Acknowledgement-This work was supported partly by the National Science Council of the Republic of China.

\section{REFERENCES}

1. Canham, L.T., Appl. Phys. Lett. 57, 1990, 1046.

2. Lockwood, D.J., Solid State Commun. 92, 1994, 101.

3. Canham, L.T., Houlton, M.R., Lerng, W.Y., Pickering, C. and Keen, J.M., J. Appl. Phys. 70, 1991, 422.

4. Tischler, M.A., Collins, R.T., Stathis, J.H. and Tsang, J.C., Appl. Phys. Lett 60, 1992, 539.

5. Chang, I.M., Pan, S.C. and Chen, Y.F., Phys. Rev. B48, $1993,8747$.

6. Xiao, Y., Heben, M.J., McCullough, J.M., Tsuo, Y.S., Pankove, J.I. and Deb, S.K., Appl. Phys. Lett. 62, 1993, 1152.

7. Stevens, P.D. and Glosser, R., Appl. Phys. Lett. 63, 1993, 803.

8. Staebler, D.L. and Wronski, C.R., J. Appl. Phys. 51, 1980, 3262.

9. Crandall, R.S., Phys. Rev. B43, 1991, 4057.

10. Chen, Y.F. and Wong, C.K., Phys. Status Solidi (b) 157, 1990, 101. 